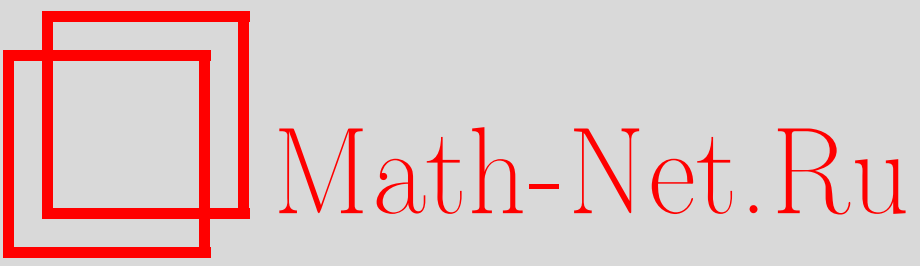

Е. И. Степанова, Суботношение Штейнера римановых многообразий, Итоги науки и техн. Сер. Соврем. мат. и ее прил. Темат. обз., 2020, том 179, 67-72

DOI: https://doi.org/10.36535/0233-6723-2020-179-67-72

Использование Общероссийского математического портала Math-Net.Ru подразумевает, что вы прочитали и согласны с пользовательским соглашением

http://www.mathnet.ru/rus/agreement

Параметры загрузки:

IP: 3.89.197.203

26 апреля 2023 г., 17:55:45 


\title{
СУБОТНОШЕНИЕ ШТЕЙНЕРА РИМАНОВЫХ МНОГООБРАЗИЙ
}

\author{
(c) 2020 г. $\quad$ Е. И. СТЕПАНОВА
}

\begin{abstract}
АннотАция. Суботношение Штейнера - одно из отношений типа Штейнера, показывающих, насколько сильно могут отличаться веса оптимальных соединяющих графов в метрическом пространстве. В статье получена верхняя оценка суботношения Штейнера римановых многообразий.
\end{abstract}

Ключевые слова: минимальная сеть Штейнера, минимальное заполнение, суботношение Штейнера.

\section{STEINER SUBRATIO OF RIEMANNIAN MANIFOLDS}

\author{
(C) 2020 E. I. STEPANOVA
}

\begin{abstract}
The Steiner subratio is one of the Steiner-type relations that show how much the weights of optimal connecting graphs in a metric space can differ. In this paper, an upper estimate for the Steiner subratio of Riemannian manifolds is obtained.
\end{abstract}

Keywords and phrases: minimal Steiner net, minimal filling, Steiner subratio.

AMS Subject Classification: 05C05, 05C22, 05C35, 53C23

1. Введение. Суботношение Штейнера - одно из трех отношений типа Штейнера, характеристик метрических пространств, которые показывают, насколько сильно в метрическом пространстве могут отличаться веса трех видов оптимальных соединяющих графов. Под графом $G$ мы понимаем пару $(V, E)$, где $V$ - конечное множество, называемое множеством вершин графа $G$, а $E$ - множество некоторых пар элементов из $V$, называемое множеством ребер графа $G$. Все необходимые определения теории графов, например, понятия степени вершины, смежных ребер, инцидентности и др., можно найти в [1].

Пусть $N$ - произвольное конечное множество и $G$-связный граф, для которого $V(G) \supset N$. Тогда будем говорить, что граф $G$ соединяет $N$. Множество графов, соединяющих $N$, обозначим через $\mathcal{G}(N)$.

Определение 1. Множество $N$ назовем множеством граничных вершин (границей) графа $G$, а множество $V(G) \backslash N$-множеством его внутренних вериин.

Замечание 1. Далее будем считать, что у всех графов имеется граница, возможно, пустая.

Ребра, инцидентные хотя бы одной граничной вершине, также будем называть граничными; остальные ребра назовем внутренними. Путь, соединяющий граничные вершины графа $G$, будем называть граничным.

Автор выражает благодарность А. О. Иванову и А. А. Тужилину за постановку задачи и внимание к работе.

Работа выполнена при поддержке Программы Президента РФ для ведущих научных школ России (проект НШ-6399.2018.1, соглашение 075-02-2018-867) и Российского фонда фундаментальных исследований (проект № 1601-00378-a). 
Определение 2. Граф $G$ называется взвешенным, если на множестве его ребер $E$ задана весовая функиия $\omega: E \rightarrow \mathbb{R}$, которая ставит в соответствие каждому ребру $e \in E$ его вес $\omega(e)$. Сумма весов всех ребер графа $G$ называется весом графа $G$ и обозначается через $\omega(G)$.

Существует несколько подходов к оптимальному соединению множества точек $N$, лежащего в метрическом пространстве $\mathbb{X}$. Несмотря на то, что понятие заполнения было введено позже понятия сети, для единообразия нам будет удобно начать именно с определения заполнения множества точек в метрическом пространстве. Оно было предложено А. О. Ивановым и А. А. Тужилиным в [2]. В общем случае заполнение не предполагает, что внутренние вершины лежат в $\mathbb{X}$. Ограничение на весовую функцию здесь возникает по аналогии с ограничением на вес минимального заполнения в задаче М. Громова (см. [8]).

Определение 3. Пара $(G, \omega)$ называется заполнением подмножества $N$ метрического пространства $\mathbb{X}$, если $G$-граф, соединяющий $N$, а $\omega$-неотрицательная весовая функция такая, что вес любого пути, соединяющего любую пару граничных вершин, не меньше расстояния между ними в метрике $\rho$ пространства $\mathbb{X}$. При этом граф $G$ называется параметризующим графом заполнения $(G, \omega)$.

Семейство всех заполнений множества $N$ будем обозначать через $\mathfrak{G}(N)$.

Определение 4. Пусть $\mathcal{K} \subset \mathfrak{G}(N)$ - некоторый класс заполнений, то есть заполнения, обладающие определенным свойством. Число

$$
\operatorname{mf}(N, \mathcal{K})=\inf _{(G, \omega) \in \mathfrak{G}(N)}\{\omega(G) \mid(G, \omega) \in \mathcal{K}\}
$$

будем называть весом минимального заполнения множества $N$ в классе $\mathcal{K}$, а каждое заполнение из $\mathcal{K}$, имеющее вес $\operatorname{mf}(N, \mathcal{K})$, называется минимальным заполнением множсества $N$ в классе $\mathcal{K}$. Минимальное заполнение $N$ в произвольном классе $\mathcal{K}$ будем называть оптимальным соединяющим графом с граничей $N$ в смысле $\mathcal{K}$ или оптимальным заполнением в классе $\mathcal{K}$.

Тривиальный класс - это все заполнения множества, без каких-либо ограничений.

Определение 5. Оптимальный соединяющий граф множества $N$ в классе всех заполнений называется минимальным заполнением множества $N$. Вес минимального заполнения множества $N$ обозначим через $\operatorname{mf}(N)$ или $\operatorname{mf}(N, \rho)$, если нам важно обозначить метрику пространства $\mathbb{X}$.

Естественный способ задать нетривиальный класс-поместить все внутренние вершины заполнения в $\mathbb{X}$ и положить вес каждого ребра равным расстоянию между его концами. Тогда веса ребер будут зависеть от положения внутренних вершин. Так получается одна из самых известных задач об оптимальном соединении точек - задача Штейнера. В ней требуется соединить $M$ графом наименьшего возможного веса так, что все его внутренние вершины лежат в $\mathbb{X}$, а вес каждого ребра равен расстоянию между концами этого ребра.

Определение 6. Сетъю с границей $N$ называется заполнение $N$, для которого определено отображение $\phi: V(G) \rightarrow \mathbb{X}$ такое, что элементы $N$ переходят в себя, а вес каждого ребра сети равен расстоянию между точками в $\mathbb{X}$, соответствующими его концам. Весовую функцию сети назовем длиной и обозначим через $\rho$.

Множество сетей с границей $N$ обозначим через $\Gamma(N)$.

Определение 7. Оптимальный соединяющий граф в классе сетей называется минимальной сетъю Штейнера. Другими словами, число

$$
\operatorname{smn}(N)=\inf _{G}\{\rho(G) \mid G \in \Gamma(N)\}
$$

называется длиной минимальной сети Штейнера, а каждая сеть, имеющая длину $\operatorname{smn}(N)$, называется минимальной сетъю Штейнера. Если мы акцентируем внимание на том, что $N$ лежит в пространстве с метрикой $\rho$, то для минимальной сети Штейнера для $N$ будем использовать обозначение $\operatorname{smn}(N, \rho)$. 
Еще один подход к заданию класса - вообще запретить добавление внутренних точек. Он ведет к задаче о поиске оптимального взвешенного графа в классе заполнений без внутренних вершин. Очевидно, наиболее выгодная весовая функция в этом случае также будет длиной. Искомый граф в этом случае - хорошо известное минимальное остовное дерево. Длина минимального остовного дерева, соединяющего множество $N$, обозначается через $\operatorname{mst}(N)$.

Далее через \# $N$ будем обозначать количество элементов в множестве $N$.

Отношения типа Штейнера показывают, насколько сильно отличаются веса оптимальных заполнений в разных классах. В 1968 году Гильберт и Поллак в [7] предложили взять отношение длины минимальной сети Штейнера к длине минимального остовного дерева. Точная нижняя грань таких отношений, взятая по всем $n$-точечным подмножествам пространства $\mathbb{X}$, называется отношением Штейнера степени $n$ пространства $\mathbb{X}$ и обозначается через $\operatorname{sr}_{n}(\mathbb{X})$. Инфимум этих отношений, взятый по всем конечным неодноточечным подмножествам $\mathbb{X}$, был назван отношением Штейнера пространства $\mathbb{X}$. Эта величина обозначается через $\operatorname{sr}(\mathbb{X})$.

Введя понятие минимального заполнения конечных метрических пространств, А. О. Иванов и А. А. Тужилин предложили рассмотреть еще два отношения. Близость минимального заполнения и минимального остовного дерева показывает отношение Штейнера-Громова.

Третье отношение называется суботношением Штейнера. Приведем его определение.

Определение 8. Величина

$$
\operatorname{ssr}(N)=\frac{\operatorname{mf}(N)}{\operatorname{mst}(N)}
$$

называется суботношением Штейнера множества $N$. Если необходимо указать метрику $\rho$ пространства $\mathbb{X}$, то будем обозначать суботношение Штейнера множества $N$ через $\operatorname{sgr}(N, \rho)$. Число

$$
\operatorname{ssr}_{n}(\mathbb{X})=\inf _{N \subset \mathbb{X}}\{\operatorname{ssr}(N) \mid 1<\# N \leqslant n\}
$$

называется суботношением Штейнера степени п пространства $\mathbb{X}$. Величина

$$
\operatorname{ssr}(\mathbb{X})=\inf _{n>1} \operatorname{ssr}_{n}(\mathbb{X})
$$

называется суботношением Штейнера пространства $\mathbb{X}$.

Заметим, что каждое отношение типа Штейнера степени 2 равно 1 для любого метрического пространства. А. О. Иванов и А. А. Тужилин в [2] показали, что суботношение Штейнера степени 3 евклидова пространства равно $\sqrt{3} / 2$, то есть равно отношению Штейнера степени 3 евклидовой плоскости. В [7] была выдвинута гипотеза, что отношение Штейнера евклидовой плоскости равно тому же числу, однако она до сих пор остается недоказанной (см. [9]). Возникло предположение, что суботношение евклидовой плоскости равно тому же числу. Однако оказалось, что максимальное число $n$, для которого суботношение плоскости степени $n$ равно $\sqrt{3} / 2$, это $4\left(\operatorname{ssr}_{4}\left(\mathbb{R}^{2}\right)\right)$ вычислено в [6]). 3. Н. Овсянников в [5] показал, что в классе выпуклых пятиугольников суботношение Штейнера все еще совпадает с четырехточечным, но $\operatorname{ssr}_{5}\left(\mathbb{R}^{2}\right)<\sqrt{3} / 2$. Там же было установлено, что $\operatorname{ssr}_{4}\left(\mathbb{R}^{3}\right)<\sqrt{3} / 2$.

В [3] А. О. Иванов и А. А. Тужилин показали, что отношение Штейнера любого связного риманова многообразия не больше, чем отношение плоскости. В [4] В. А. Мищенко доказала аналогичный результат для отношения Штейнера-Громова. В настоящей работе, используя аналогичную технику, мы покажем, что для любого связного риманова многообразия суботношение Штейнера не больше, чем суботношение плоскости, а значит, с учетом приведенных выше результатов, оно меньше $\sqrt{3} / 2$.

\section{2. Основные результаты.}

Теорема 1. Суботношение Штейнера произвольного связного риманова многообразия размерности $n$ не превосходит суботношения Штейнера евклидова пространства $\mathbb{R}^{n}$.

Сначала докажем несколько вспомогательных результатов. 
Лемма 1. Пусть $X-$ множество, а $\rho_{1}$ и $\rho_{2}$-метрики на нем. Предположим, что для некоторых чисел $c_{2} \geqslant c_{1}>0$ неравенства $c_{1} \rho_{2}(x, y) \leqslant \rho_{1}(x, y) \leqslant c_{2} \rho_{2}(x, y)$ выполнены для любых mочек $x$ и у из $X$. Тогда

$$
\frac{c_{1}}{c_{2}} \operatorname{ssr}\left(X, \rho_{2}\right) \leqslant \operatorname{ssr}\left(X, \rho_{1}\right) \leqslant \frac{c_{2}}{c_{1}} \operatorname{ssr}\left(X, \rho_{2}\right) .
$$

Доказательство. Если $G$-минимальная сеть Штейнера с границей $N \subset X$ в метрике $\rho_{1}$, то $G$ является соединяющей $N$ сетью в любой другой метрике, поэтому

$$
\operatorname{smn}\left(N, \rho_{1}\right)=\rho_{1}(G) \geqslant c_{1} \rho_{2}(G) \geqslant c_{1} \operatorname{smn}\left(N, \rho_{2}\right) .
$$

Аналогично

$$
\operatorname{smn}\left(N, \rho_{2}\right) \geqslant \frac{1}{c_{2}} \operatorname{smn}\left(N, \rho_{1}\right) \Longleftrightarrow \operatorname{smn}\left(N, \rho_{1}\right) \leqslant c_{2} \operatorname{smn}\left(N, \rho_{2}\right) .
$$

В [4] было показано, что $\operatorname{mf}\left(N, \rho_{1}\right) \geqslant c_{1} \operatorname{mf}\left(N, \rho_{2}\right)$ и $\operatorname{mf}\left(N, \rho_{1}\right) \leqslant c_{2} \operatorname{mf}\left(N, \rho_{2}\right)$. Поэтому для любого конечного неодноточечного множества $N$, для которого существует минимальная сеть Штейнера (минимальное заполнение существует всегда; см. [2]),

Значит,

$$
\operatorname{ssr}\left(N, \rho_{1}\right)=\frac{\operatorname{mf}\left(N, \rho_{1}\right)}{\operatorname{smn}\left(N, \rho_{1}\right)} \leqslant \frac{c_{2} \operatorname{mf}\left(N, \rho_{2}\right)}{c_{1} \operatorname{smn}\left(N, \rho_{2}\right)}=\frac{c_{2}}{c_{1}} \operatorname{ssr}\left(N, \rho_{2}\right) .
$$

$$
\operatorname{ssr}\left(X, \rho_{1}\right)=\inf _{N} \operatorname{ssr}\left(N, \rho_{1}\right) \leqslant \frac{c_{2}}{c_{1}} \inf _{N} \operatorname{ssr}\left(N, \rho_{2}\right)=\frac{c_{2}}{c_{1}} \operatorname{ssr}\left(X, \rho_{2}\right) .
$$

Аналогично получается второе неравенство из утверждения леммы.

Пусть $(X, \rho)$ - метрическое пространство и $\left(X^{\prime}, \rho^{\prime}\right)$ - его подпространство, т. е. $X^{\prime} \subset X$ и для любых точек $u, v \in X^{\prime}$ выполнено $\rho^{\prime}(u, v)=\rho(u, v)$. Так как каждое конечное подмножество подпространства $X^{\prime}$ является также конечным подмножеством всего пространства $X$, справедливо следующее утверждение.

Лемма 2. Пусть $(X, \rho)$-метрическое пространство, $\left(X^{\prime}, \rho^{\prime}\right)$ - его подпространство. Тогда $\operatorname{ssr}\left(X^{\prime}, \rho^{\prime}\right) \geqslant \operatorname{ssr}(X, \rho)$.

Следующая лемма основана на том, что оптимальный соединяющий граф останется соединяющим при отображении, не увеличивающем расстояния. Доказательство можно найти в $[3,4]$.

Лемма 3. Пусть $f: X \rightarrow Y$-отображение метрического пространства $\left(X, \rho_{X}\right)$ в метрическое пространство $\left(Y, \rho_{Y}\right)$, не увеличивающее расстолний, то есть $\rho_{X}(x, y) \geqslant \rho_{Y}(f(x), f(y))$ для любых точек $x$ и у из $X$. Тогда для любого конечного подмножества $N \subset X$ выполняется:

$$
\operatorname{mf}(N) \geqslant \operatorname{mf}(f(N)), \quad \operatorname{smn}(N) \geqslant \operatorname{smn}(f(N)) .
$$

Пусть $M$ - связное $n$-мерное риманово многообразие. Для любой кусочно-гладкой кривой $\gamma$ ее длину по отношению к римановой метрике обозначим через $\operatorname{len}(\gamma)$. Тогда внутренняя метрика $\rho$, порожденная римановой метрикой, - это точная нижняя грань длин всех таких кривых, соединяющих точки, то есть $\rho(x, y)=\inf _{\gamma} \operatorname{len}(\gamma(x, y))$, где $\gamma(x, y)$ - произвольная кусочно-гладкая кривая, соединяющая точки $x$ и $y$ на $M$.

Пусть $P$-точка многообразия $M$. Рассмотрим нормальные координаты $x_{1}, \ldots, x_{n}$ с центром в точке $P$, для которых риманова метрика $g_{i j}$, вычисленная в точке $P$, совпадает с $\delta_{i j}$. Возьмем выпуклый открытый шар $U(\delta)$ с центром в точке $P$ радиуса $\delta$, для которого каждая пара его точек $x$ и $y$ соединяется единственной кратчайшей геодезической $\gamma$, лежащей в $U(\delta)$. Тогда $\rho(x, y)=$ $\operatorname{len}(\gamma)$. Таким образом, $U(\delta)$ является метрическим пространством с внутренней метрикой.

В координатах $x_{1}, \ldots, x_{n}$ шар $U(\delta)$ задается так: $U(\delta)=\left\{x_{1}^{2}+x_{2}^{2}+\ldots+x_{n}^{2}<\delta^{2}\right\}$, поэтому если на $U(\delta)$ задать евклидово расстояние $\rho_{e}$ (по отношению к нормальным координатам $x_{i}$ ), то метрическое пространство $\left(U(\delta), \rho_{e}\right)$ также будет пространством с внутренней метрикой, порожденной евклидовой метрикой $\delta_{i j}$.

В силу гладкой зависимости метрики $g_{i j}(x)$ от точки $x$ в шаре $U(\delta)$, для любого положительного числа $\varepsilon<\frac{1}{n^{2}}$ существует такое положительное число $\delta$, что для всех точек $x$ из $U(\delta)$ выполнено:

$$
\left|g_{i j}(x)-\delta_{i j}\right|<\varepsilon \text {. }
$$


Отсюда вытекает следующая лемма, доказательство которой приведено в [3].

Лемма 4. Пусть $\|v\|_{g}$ обозначает длину касательного вектора $v \in T_{x} M$ в метрике $g_{i j}$, a $\|v\|_{e}-$ дину этого вектора в евклидовой метрике $\delta_{i j}$. Если для любых $i$ и $j$ выполняется неравенство (1), то

$$
\sqrt{1-n^{2} \varepsilon}\|v\|_{e} \leqslant\|v\|_{g} \leqslant \sqrt{1+n^{2} \varepsilon}\|v\|_{e}
$$

Опираясь на определение расстояния между точками связного риманова многообразия, сразу же получаем следующий результат.

Следствие 1. Пусть $M$ - произвольное связное риманово многообразие, $U(\delta)$ - открытый шар радиуса $\delta, \rho$ - внутренняя метрика, порожденная римановой метрикой на нем, $\rho_{e}-$ внутренняя метрика, порожденная евклидовой метрикой на нем. Тогда для любого положительного $\varepsilon<\frac{1}{n^{2}}$ существует положительное $\delta$ такое, что для всех точек $x, y \in U(\delta)$ выполняется

$$
\sqrt{1-n^{2} \varepsilon} \rho_{e}(x, y) \leqslant \rho(x, y) \leqslant \sqrt{1+n^{2} \varepsilon} \rho_{e}(x, y)
$$

Лемма 5. Пусть $U$-открытый шар в $\mathbb{R}^{n}$ со стандартной евклидовой метрикой. Тогда $\operatorname{ssr}\left(\mathbb{R}^{n}\right)=\operatorname{ssr}(U)$.

Доказательство. Любое конечное множество $N$ можно сжатием и сдвигом поместить в шар $U$, при этом все расстояния умножаются на одно и то же число $\lambda$. Поэтому и вес каждого оптимального соединяющего графа умножится на $\lambda$, а значит, все отношения типа Штейнера сохранятся, откуда и следует утверждение леммы.

Из всего сказанного вытекает следующий результат.

Следствие 2. Пусть $M$-произвольное связное риманово многообразие размерности $n$ u $U(\delta)$-открытый шар радиуса $\delta$ с иентром в некоторой точке $P \in M$. Обозначим через $\rho$ внутреннюю метрику на $M$, порожденную римановой метрикой. Тогда для любого положительного числа $\varepsilon<\frac{1}{n^{2}}$ существует положительное $\delta$ такое, что для всех точек $x$ и из $U(\delta)$ верно:

$$
\sqrt{\frac{1-n^{2} \varepsilon}{1+n^{2} \varepsilon}} \operatorname{ssr}\left(\mathbb{R}^{n}\right) \leqslant \operatorname{ssr}(U(\varepsilon), \rho) \leqslant \sqrt{\frac{1+n^{2} \varepsilon}{1-n^{2} \varepsilon}} \operatorname{ssr}\left(\mathbb{R}^{n}\right) .
$$

Доказательство теоремъ 1. Пусть $M$ - связное риманово $n$-мерное многообразие, $\rho-$ внутренняя метрика на нем, порожденная римановой метрикой. Пусть $P$ - произвольная точка из $M$ и $U(\delta)$ - открытый шар радиуса $\delta$ с центром в этой точке. Введем в $U(\delta)$ нормальные координаты $x_{i}$, и пусть $\rho_{e}$ - внутренняя метрика на $U(\delta)$, порожденная евклидовой метрикой $\delta_{i j}$. Здесь $\delta$ такое же, как в следствии 2.

Пусть $\left\{\varepsilon_{i}\right\}$ - некоторая убывающая последовательность положительных чисел такая, что $\varepsilon_{i}<$ $\delta$ для любого $i$ и $\varepsilon_{i} \rightarrow 0$ при $i \rightarrow \infty$. Рассмотрим семейство вложенных множеств $X_{i}=U\left(\varepsilon_{i}\right)$. Из леммы 5 получаем, что $\operatorname{ssr}\left(U\left(\varepsilon_{i}\right), \rho_{e}\right)=\operatorname{ssr}\left(\mathbb{R}^{n}\right)$.

В силу выпуклости шаров $U\left(\varepsilon_{i}\right)$, внутренняя метрика на них, порожденная римановой метрикой многообразия $M$, совпадает с ограничением метрики $\rho$. По следствию 2 ,

$$
\operatorname{ssr}\left(X_{i}\right) \leqslant \sqrt{\frac{1+n^{2} \varepsilon}{1-n^{2} \varepsilon}} \operatorname{ssr}\left(\mathbb{R}^{n}\right)
$$

Так как подкоренное выражение стремится к 1 при $i \rightarrow \infty$, то $\inf _{i} \operatorname{ssr}\left(X_{i}, \rho\right) \leqslant \operatorname{ssr}\left(\mathbb{R}^{n}\right)$, но по лемме $2 \operatorname{ssr}(M, \rho) \leqslant \inf _{i} \operatorname{ssr}\left(X_{i}, \rho\right)$.

Доказательство окончено.

\section{СПИСОК ЛИТЕРАТУРЫ}

1. Емеличев В. А., Мельников О. И., Сарванов В. И., Тышкевич Р. И. Лекции по теории графов. - М.: Наука, 1990.

2. Иванов A. О., Тужилин А. А. Одномерная проблема Громова о минимальном заполнении// Мат. сб. -2012 . -203 , № 5. - C. $65-118$. 
3. Иванов А. О., Тужилин А. А., Цислик Д. Отношение Штейнера для многообразий// Мат. заметки. 2003. - 74, № 3. - С. 387-395.

4. Мищенко B. А. Оценки отношения Штейнера-Громова римановых многообразий// Фундам. прикл. мат. - 2013. - 18, № 2. - С. 119-124.

5. Овсянников 3. Н. Суботношение Штейнера для пяти точек на плоскости и четырех точек в пространстве// Фундам. прикл. мат. - 2013. - 18, № 2. - С. 167-179.

6. Степанова Е. И. Бифуркации минимальных деревьев Штейнера и минимальных заполнений для невыпуклых четырехточечных границ и суботношение Штейнера евклидовой плоскости// Вестн. Моск. унта. Cер. 1. Мат. Мех. - 2016. - № 2. - C. 48-51.

7. Gilbert E. N., Pollak H. O. Steiner minimal trees// SIAM J. Appl. Math. — 1968. — 16, № 1. - P. 1-29.

8. Gromov M. Filling Riemannian manifolds// J. Differ. Geom. — 1983. — 18, № 1. — P. 1-147.

9. Ivanov A. O., Tuzhilin A. A. The Steiner ratio Gilbert-Pollak conjecture is still open// Algorithmica. 2012. - 62, № 1. - P. 630-632.

Степанова Екатерина Ивановна

Московский государственный университет имени М. В. Ломоносова

E-mail: ekfila@gmail.com 